\title{
Genus-specific kinetoplast-DNA PCR and parasite culture for the diagnosis of localised cutaneous leishmaniasis: applications for clinical trials under field conditions in Brazil
}

\author{
Julia Ampuero, Alexandre Pereira Rios, César Omar Carranza-Tamayo, Gustavo Adolfo Sierra Romero/ ${ }^{+}$ \\ Núcleo de Medicina Tropical, Universidade de Brasília, Campus Universitário Darcy Ribeiro, Caixa postal 4517, 70904-970 Brasília, DF, Brasil
}

The positivities of two methods for the diagnosis of localised cutaneous leishmaniasis (CL) were estimated in 280 patients enrolled in a clinical trial. The trial was conducted in an endemic area of Leishmania (Viannia) braziliensis and trial participants were patients with skin ulcers and positive leishmanin skin tests. Patients underwent aspirative skin punctures of the ulcerated lesions and lymph nodes for in vitro cultures, which were processed under field conditions at the local health centre. Skin lesion biopsies were tested at a reference laboratory using kinetoplastid $D N A$ ( $k D N A)-P C R$ to detect DNA. The median time required to obtain a positive culture from the skin samples was seven days and the contamination rate of the samples was $1.8 \%$. The positivities of the cultures from skin lesions, kDNA-PCR and the combination of the two methods were $78.2 \%$ (95\% CI: 73-82.6\%), 89.3\% (95\% CI: 85.1-92.4\%) and $97.1 \%$ (95\% CI: 94.5-98.5\%). We conclude that parasite culture is a feasible method for the detection of Leishmania in field conditions and that the combination of culture and PCR has a potential role for the diagnosis of CL in candidates for clinical trials.

Key words: cutaneous leishmaniasis - Leishmania braziliensis - polymerase chain reaction - isolation and purification - clinical trials

Cutaneous leishmaniasis (CL) is caused by protozoa belonging to the genus Leishmania and the disease is a widespread public health problem that mainly affects low-income populations (Grimaldi Jr \& Tesh 1993, Murray et al. 2005, Alvar et al. 2006). Between 19852005 , the mean annual number of autochthonous cases was 28,568 , as reported to the Brazilian surveillance system; this corresponds to a mean detection coefficient of 18.5 cases $/ 100,000$ inhabitants. The highest incidence rate was observed in the North and Northeast Regions of Brazil, with most of the cases registered in the states of Maranhão, Ceará and Bahia (BA). The cases registered in BA were detected in the Southern Region, where the Corte de Pedra Health Centre is located. In that region, most cases of leishmaniasis are caused by Leishmania (Viannia) braziliensis (Rosa et al. 1988, Romero et al. 2001c).

It is possible to observe relevant differences in clinical presentation, diagnostic assay sensitivity and response to specific therapeutics based on the species of Leishmania causing the infection (Romero et al. 2001 a, c, Arévalo et al. 2007). Parasitological tests, including the direct observation of amastigotes in scrapings from skin lesions, cultures and the inoculation of tis-

Financial support: Secretaria de Vigilância em Saúde do Ministério da Saúde, FAPDF (PRONEX/FAPDF 193.000-114/2004), FINATEC, $\mathrm{PIBIC} / \mathrm{CNPq} / \mathrm{MCT}, \mathrm{CAPES} / \mathrm{MEC}$

+ Corresponding author: gromero@unb.br

Received 18 May 2009

Accepted 2 September 2009 sue samples into hamsters, have usually demonstrated low sensitivity for the diagnosis of $L$. (V.) braziliensis infections. This problem was not observed to the same magnitude in infections caused by Leishmania (Viannia) guyanensis (Romero et al. 2001c). The main disadvantages related to conventional parasitological methods include the need for qualified technicians to perform the collection, preparation, preservation and observation of the samples under field conditions, as well as in reference laboratories. Furthermore, cultures have the additional disadvantage of potential contamination with fungi or bacteria, which has been associated with prolonged observation (Cuba Cuba et al. 1986a, b). In recent years, the collection method has been optimised to reduce the problem of contamination and to increase the sensitivity of the diagnostic methods (Marzochi et al. 1993, Romero et al. 1999a, b). Recently, the use of molecular diagnostic tools such as PCR assays specific to different DNA targets has increased (Marques et al. 2001, Safaei et al. 2002, Schallig \& Oskam 2002, Weigle et al. 2002). The amplification of the conserved sequences found in the minicircles of kinetoplastid DNA ( $\mathrm{kDNA}$ ) of Leishmania using PCR is currently one of the most common and available techniques (Pirmez et al. 1999, Bensoussan et al. 2006).

The lack of sensitivity and specificity of the diagnostic methods that can currently be used in rural areas poses a large challenge to conducting clinical trials with patients affected by leishmaniasis in these areas. Although parasite culture has been considered by some authors (Reithinger et al. 2007) to be the gold standard due to its high specificity, the problem of its low sensitivity remains unresolved (Murray et al. 2005, Tojal da Silva et al. 2006). In the absence of a true gold standard diagnostic assay for $\mathrm{CL}$, it is likely that a combination of disease-defining 
characteristics more accurately represents the true disease status (Coggon et al. 2005). This approach was used in the present paper to establish the positivity of the methods under evaluation using a case definition composed of skin ulcers considered suspicious of being caused by leishmaniasis plus a positive leishmanin skin test.

The present paper was undertaken to estimate the positivity of in vitro cultures of samples obtained through aspirative punctures from the cutaneous ulcer borders of skin lesions. These biopsies were conducted in a rural health centre and PCR was performed on the samples at a reference laboratory to detect Leishmania DNA using kDNA-PCR for the diagnosis of localised CL.

\section{PATIENTS AND METHODS}

Study type and patients - This study examined the accuracy of two diagnostic tests for leishmaniasis and was nested in a clinical trial designed to evaluate the effectiveness and safety of low doses of pentavalent antimonials for the treatment of CL caused by $L$. (V.) braziliensis (ClinicalTrials.gov NCT00317980). Patients were considered as candidates if they presented with skin ulcers suspicious of leishmaniasis (painless ulcerated lesions with raised borders and granulose bottoms) and a positive leishmanin skin test. All of the patients in the study were treated for leishmaniasis at the local health centre located at Corte de Pedra District, Presidente Tancredo Neves municipality, BA, Brazil, between January 2006-January 2007. Two hundred-eighty patients were enrolled in this study. The trial included male and female patients aged 7-50 years with up to nine ulcerated cutaneous lesions, a positive leishmanin skin test and not more than 20 weeks of disease evolution.

Leishmanin skin test - The skin test was performed with an antigen prepared at the Immunology Service of the Federal University of Bahia, Brazil, as previously described (Reed et al. 1986). Briefly, $0.1 \mathrm{~mL}$ of soluble components of Leishmania (Leishmania) amazonensis promastigotes, MHOM/BR/1986/BA125 strain, containing $250 \mu \mathrm{g} / \mathrm{mL}$ protein were injected intradermally on the volar surface of the left forearm and the reaction was measured after 48-72 $\mathrm{h}$ using the standard procedure (Sokal 1975). Diameters of induration greater than or equal to $5 \mathrm{~mm}$ were considered positive.

Culture medium production and transport conditions - The culture medium was prepared in the Leishmaniasis Laboratory of the Tropical Medicine Unit at the University of Brasilia using blood agar base, code 0045-17, pH 6.8 (DIFCO Ltd, Brazil) and 15\% defibrinated rabbit blood with $100 \mu \mathrm{g} / \mathrm{mL}$ gentamicin (Garamicina ${ }^{\circledR}$, ScheringPlough, Rio de Janeiro, Brazil). The tubes containing the culture medium were transported by air from Brasilia to the capital of BA, on a flight of approximately $1 \mathrm{~h}$ and 15 min duration and they were then transported by car to the Corte de Pedra District, which is approximately a $4 \mathrm{~h}$ trip. The transportation temperature was between $2-8^{\circ} \mathrm{C}$.

Culture procedures - The procedures were conducted after local cleansing of the site with $0.9 \%$ sodium chloride and then a solution with $1 \%$ of active iodine. The biopsies were performed under local anaesthesia induced with $2 \%$ lidocaine without a vasoconstrictor, according to the vacuum aspiration technique previously reported (Romero et al. 1999b). Before the aspirative procedure, $0.2 \mathrm{~mL}$ of $0.9 \%$ saline solution plus 100 $\mu \mathrm{g} / \mathrm{mL}$ gentamicin (Garamicina ${ }^{\circledR}$, Schering-Plough, Rio de Janeiro, Brazil) were added to the solid medium and the vacuum was subsequently restored using a $20 \mathrm{~mL}$ syringe. Three aspirative punctures from the elevated border of the most recent onset cutaneous lesion and three aspirative punctures from enlarged satellite lymph nodes, if present, were taken from each patient. The lymph node aspiration was performed for patients with lymph nodes larger than $3 \mathrm{~cm}$ in diameter. The aspirated material of each puncture was inoculated in separated culture tubes. The cultures were kept in the rural health centre inside of a glass closet in an air-conditioned room with the temperature maintained between $22-24^{\circ} \mathrm{C}$. Examination of the cultures was performed every $72-96 \mathrm{~h}$ using a Carl Zeiss Jena inverted microscope (Germany). Cultures were later transported to the Brasilia reference laboratory for quality control after cultures turned positive during the observation period or after four weeks of observation of negative cultures.

$k D N A-P C R$ - Immediately after the aspirative punctures, a $4 \mathrm{~mm}$ sterile punch biopsy was performed. After the removal of excess blood with filter paper, the biopsy fragment was placed inside a $2 \mathrm{~mL}$ dry polypropylene tube and maintained at $-20^{\circ} \mathrm{C}$ in the endemic area until it was transported to the reference laboratory in Brasilia. All samples were transported to Brasilia at the end of the enrolment period. DNA extraction was performed using the commercial illustra tissue \& cells genomic Prep Mini Spin $\mathrm{Kit}^{\mathrm{TM}}$ (USA) following the manufacturer's recommendations. Three primers were used to amplify the conserved region of 120 base pairs of the minicircle DNA, one forward: 5'GGG GAG GGG CGT TCT GCG AA 3' $(\mathrm{MW}=6602.0 \mu \mathrm{g} / \mu \mathrm{mol})$ and two backward: 5'CCG CCC CTA TTT TAC ACC AAC CCC 3' $(\mathrm{MW}=$ $7504.8 \mu \mathrm{g} / \mu \mathrm{mol})$ and 5'GGC CCA CTA TAT TAC ACC AAC CCC 3' (MW = 7577.8 $\mu \mathrm{g} / \mu \mathrm{mol})$. The two backward primers were aimed to accommodate sequence variation in the minicircle population. These primers have been extensively used in Brazil for genus-specific diagnosis of Leishmania infection (Degrave et al. 1994a, b). Authors have described those primers with different names and notations (Pirmez et al. 1999, Romero et al. 2001a, 2009, Volpini et al. 2004, De Assis et al. 2008, Luz et al. 2009). The amplification procedure was performed in a Perkin Elmer GeneAmp PCR System 2400 thermocycler (The Perkin Elmer Corporation, Norwalk, CT, USA). Briefly, reaction mixtures were prepared in a $10 \mu \mathrm{L}$ volume using $1 \mu \mathrm{L}$ of DNA sample in $10 \mathrm{mM}$ Tris- $\mathrm{HCl} \mathrm{pH}$ 8.6, 0.25 units of Taq DNA polymerase (Promega, Madison, WI, USA), $50 \mathrm{mM} \mathrm{KCl} \mathrm{pH} \mathrm{8.3,}$ $1.5 \mathrm{mM} \mathrm{MgCl}_{2}$ and $50 \mu \mathrm{M}$ of each dNTP. The primer concentration in the mixture was $1.2 \mu \mathrm{M}$ (forward) and $0.6 \mu \mathrm{M}$ (each backward). Thirty-nine amplification cycles were undertaken using a temperature of $95^{\circ} \mathrm{C}$ for denaturation, $66^{\circ} \mathrm{C}$ for annealing and $72^{\circ} \mathrm{C}$ for extension, with $30 \mathrm{~s}$ for each step. Five minutes of initial de- 
naturation and 5 min final extension were applied. All of the reactions were performed with appropriate positive and negative controls. The positive control was prepared with DNA extracted from a log phase culture of promastigotes of $L$. (V.) braziliensis MHOM/BR/75/M2904 with an analytic sensitivity of at least one parasite. The amplified products were then analysed by $8 \%$ polyacrylamide gel electrophoresis performed in $1 \mathrm{X}$ TBE buffer at 100 volts (Sambrook et al. 2001) and subsequent silver staining (Bassam et al. 1991).

Statistical analysis - The analysis was performed using SPSS 16.0 for Windows (SPSS Inc, Chicago, IL, USA). The proportions of positive results were calculated for each diagnostic technique along with their respective $95 \%$ confidence intervals $(95 \% \mathrm{CI})$ and proportions were compared using a Chi-squared test. Continuous, normally distributed data were stated as means plus one standard deviation. Continuous data without a normal distribution were described as medians and the 25th $\left(\mathrm{Q}_{25}\right)$ and 75th $\left(\mathrm{Q}_{75}\right)$ percentiles, respectively, were noted. Continuous variables with a normal distribution were compared using the Student's $t$-test; otherwise, the Mann-Whitney U test was applied. P values $<0.05$ were considered statistically significant.

Ethics - The clinical trial was approved by the Ethical Committee of the Faculty of Medicine of the University of Brasilia (007/2004). The research protocol was conducted in agreement with the Helsinki Declaration and Resolutions 196/1996 and 251/1997 of the National Health Council of the Brazilian Ministry of Health. All diagnostic procedures in the present study are recognised by the Brazilian Ministry of Health as valid alternatives for CL diagnosis. All patients or their legal guardians signed the informed consent paperwork before any procedures were performed.

\section{RESULTS}

In total, 280 patients were included and $67.1 \%$ were males. The median age was 21 years $\left(\mathrm{Q}_{25}-\mathrm{Q}_{75}: 15-31\right)$, the median disease duration was 30 days $\left(\mathrm{Q}_{25}-\mathrm{Q}_{75}: 28-60\right)$, $33 \%$ of the patients had lesions on their legs, $63 \%$ had only one cutaneous lesion and $94.6 \%$ of the patients presented with enlarged lymph nodes that were detected at the time of clinical diagnosis.

Of the 280 patients, 279 each received three aspirative punctures of the ulcer borders of their cutaneous lesions; the aspirative punctures subsequently underwent parasite culture. The remaining patient only received one aspirative puncture, which was then cultured. Sixty patients were willing to provide aspirative cultures of their satellite lymph nodes. Consequently, 838 culture tubes inoculated with skin ulcer samples and 180 culture tubes inoculated with lymph node samples were observed as previously described.

The positivity of the culture tubes inoculated with skin samples was $65.8 \%$ (551/838): 190 of 280 tubes $(67.9 \%)$ inoculated with the first aspirative puncture sample, 186 of 279 tubes (66.7\%) inoculated with the second sample and 175 of 279 tubes $(62.7 \%)$ inoculated with the third sample were found to be positive. The me- dian time required to obtain a positive culture tube from a skin sample was seven days $\left(\mathrm{Q}_{25}-\mathrm{Q}_{75}: 5-10\right)$ (Figure), with the time required ranging from 1-31 days. Fungal contamination was found in $1.8 \%$ of skin culture tubes.

The overall positivity of the culture tubes inoculated with the lymph node samples was $36.6 \%$ (66/180): 20 of $60(33.3 \%)$ inoculated with the first aspirative puncture sample, 25 of $60(41.7 \%)$ inoculated with the second sample and 21 of 60 tubes $(35 \%)$ inoculated with the third sample were found to be positive. The median time required to obtain a positive culture tube after inoculation with the lymph node samples was seven days $\left(\mathrm{Q}_{25}-\mathrm{Q}_{75}\right.$ : 6-10; range 3-27 days) (Figure). Fungal contamination was found in $2.2 \%$ of lymph node culture tubes. Of note, two patients who presented with negative skin cultures were found to have positive lymph node cultures.

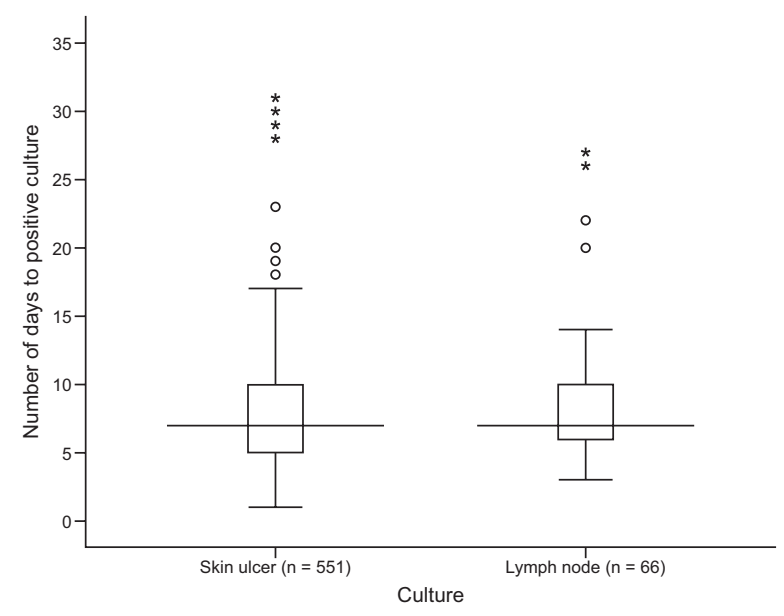

Distribution of the number of days to obtain a positive culture around the median (horizontal line) depending on the sample source in patients with cutaneous leishmaniasis. Boxes extend from 25-75th percentiles. Whiskers extend to the largest and smallest observed values in the distribution having the limits which correspond to 1.5 times the box length around the median. Circles represent outliers and asterisks extreme values.

Of patients who had positive skin cultures collected, $65 \%(143 / 219)$ had positive results in all three culture tubes inoculated with skin samples, $21 \%(46 / 219)$ had positive results in two tubes and $13.7 \%(30 / 219)$ had only one positive sample. Conversely, 14 of the 27 patients with positive cultures from lymph node punctures had positive results from all three samples, 11 patients had two positive samples and two patients had only one positive sample.

The overall positivity of the skin cultures, lymph node cultures and both types of cultures combined, considering the patient as the unit for this analysis, is shown in Table I.

Positivity of the kDNA-PCR was $89.3 \%$. Combining both diagnostic methods increased the overall positivity to $97.1 \%$ (Table I). Overall, eight patients had no demonstration of the presence of Leishmania by the two studied methods. 


\section{TABLE I}

Positivity of diagnostic methods for cutaneous leishmaniasis in patients with clinical suspected ulcers and a positive leishmanin skin test

\begin{tabular}{|c|c|c|c|}
\hline Method & $\begin{array}{l}\text { Patients with positive results/ } \\
\text { patients examined } \\
n\end{array}$ & $\begin{array}{c}\text { Positivity } \\
\%\end{array}$ & $95 \% \mathrm{CI}$ \\
\hline Skin culture (any culture tube) & $219 / 280$ & 78.2 & $73.0-82.6$ \\
\hline Lymph node culture (any culture tube) & $27 / 60$ & 45.0 & $33.1-57.5$ \\
\hline Skin or lymph node culture (any culture tube) & $221 / 280$ & 78.9 & $73.8-83.3$ \\
\hline Kinetoplastid DNA-PCR & $250 / 280$ & 89.3 & $85.1-92.4$ \\
\hline Skin or lymph node culture or kinetoplastid DNA-PCR & $272 / 280$ & 97.1 & $94.5-98.5$ \\
\hline
\end{tabular}

CI: confidence interval.

Using skin lesion culture as the gold standard, the sensitivity of the kDNA-PCR assay, evaluated using the data of the 219 patients with positive cultures, was $90 \%$ (95\% CI: 85.4-93.4\%). The agreement between the culture and PCR results is demonstrated in Table II.

\section{DISCUSSION}

Conducting clinical trials in health centres located in rural areas where leishmaniasis is endemic represents a challenge for researchers because of the difficulty associated with confirming the diagnosis. Ideally, methods to confirm the diagnosis should be easy to use and capable of producing rapid results, allowing the crucial decision of patient inclusion in a trial to be made. To maintain the scientific rigor of the research, it is necessary to obtain parasitological confirmation of each case to maintain high internal validity of the study and to avoid deleterious exposure of patients without leishmaniasis to the drugs under evaluation. The present paper was not intended to validate the diagnostic methods but to demonstrate that a combination of parasite culture available under field conditions and molecular tools applied in samples sent to a reference lab could be a useful and viable solution for parasitological diagnosis of human cases in clinical trials.

Most cases of leishmaniasis in the endemic area of Corte de Pedra are caused by L. (V.) braziliensis. Therefore, it offers an ideal setting for clinical research and the evaluation of diagnostic tests for the diseases caused by this species without the confusion derived from the sympatric circulation of other species of Leishmania (Rosa et al. 1988, Romero et al. 2001c, 2009). Early studies conducted in the areas of Três Braços and Corte de Pedra performed cultures from skin lesions, obtained positivity rates of $15.3-30.8 \%$ and had a contamination rate as high as $27.5 \%$. Positivity increased to $51 \%$ when three cultures were obtained from each patient and each aspirate came from three different points along the ulcer border of the same lesion (Cuba Cuba et al. 1986a). We suggested in two previous reports with a small sample of patients (Romero et al. 1999a, b) that the combined approach of aspirative punctures of skin ulcers and lymph nodes produces better results. The present research, which evalu-
TABLE II

Comparison of the results obtained with cultures from skin ulcers and the kinetoplastid DNA-PCR (kDNA-PCR) assay in patients with cutaneous ulcers suspected of leishmaniasis and a positive leishmanin skin test

\begin{tabular}{lccc}
\hline & \multicolumn{2}{c}{$\begin{array}{c}\text { Isolation of Leishmania } \\
\text { from skiculture }\end{array}$} & \\
\cline { 2 - 3 } & $\begin{array}{c}\text { Positive } \\
\mathrm{n}(\%)\end{array}$ & $\begin{array}{c}\text { Negative } \\
\mathrm{n}(\%)\end{array}$ & Total \\
\hline Result of the kDNA-PCR & $197(90)$ & $53(86.9)$ & 250 \\
Positive & $22(10)$ & $8(13.1)$ & 30 \\
Negative & $219(100)$ & $61(100)$ & 280 \\
Total & & & \\
\hline
\end{tabular}

ated a substantially higher number of patients, did not confirm the findings of our previous reports, but the low receptivity of the patients allowing the lymph node puncture. The overall skin culture and PCR yield was similar to those reported by other authors (Luz et al. 2009), which confirmed the reproducibility of this method. The low contamination rate was also reproduced in the present study with a larger number of observations.

It is known that the positivity of parasitological diagnostic methods is directly associated with the duration of the presence of the lesion. In our research, the median duration of cutaneous disease was 30 days, which might have contributed to the performance of the culture. The modification of culture methods to accelerate the time necessary to obtain positive results has been reported, with sensitivities ranging from $83-97 \%$ in Turkey (Allahverdiyev et al. 2004) and from $71.7-84.7 \%$ in Peru, where the investigators used microcultures and minicultures in polypropylene tubes (Boggild et al. 2007, 2008).

Some molecular methods have elevated specificity for diagnosing leishmaniasis and have the advantage of identification of parasite species, strains and even clones. However, those more specific methods are the least sensitive and work better when applied for the identification of isolated parasites obtained from cultures (Schallig \& 
Oskam 2002, Bensoussan et al. 2006, Tojal da Silva et al. 2006). The use of PCR to amplify the conserved 120-bp region of kDNA has been shown to be the most sensitive approach for routine diagnostic use (Tojal da Silva et al. 2006). The sensitivity is higher than other assays because it can accurately detect cases of leishmaniasis when the identification of parasite species is not essential. The combination of different molecular approaches allows appropriate parasite identification in areas where sympatric circulation of several Leishmania species is a problem (Bensoussan et al. 2006, Tojal da Silva et al. 2006). Our study demonstrated a PCR positivity of $89 \%$, which is lower than has been described in other areas (Pirmez et al. 1999, Gomes et al. 2008, Reis et al. 2008). However, our results are consistent with a previous study in the same area that reported a positivity of $88.3 \%$ for PCR performed with DNA obtained from fresh-frozen lesion biopsies and positivities of $87 \%$ and $85.7 \%$ for PCR performed with DNA obtained from biopsy imprints on filter paper in two independent laboratories (Romero et al. 2009). This consistency between results obtained during different periods allows us to suggest that in the area studied, the two diagnostic methods that were performed in the present trial should be combined for the diagnostic confirmation of Leishmania infection.

The challenge for the study of the validity of diagnostic tests for leishmaniasis is the lack of a gold standard. Parasite culture has low sensitivity and when used as the gold standard, it causes artificially low estimates for specificity due to the increase in "false-positive" results. This was the case in our study, as PCR detected $86.9 \%$ of the culture-negative cases. On the other hand, PCR is not a perfect new tool, because it did fail to detect Leishmania in $10 \%$ of the culture-positive cases.

The observation of cultures in the endemic area, the use of methods of sample conservation for performing PCR using DNA obtained from imprints on filter paper (Marques et al. 2001, Romero et al. 2009) and improvement of fresh biopsy tissue transportation (Castilho et al. 2003) allow initiatives to develop clinical research under field conditions, as these methods do not require sophisticated equipment to obtain an early diagnosis. Sample transportation for processing in reference laboratories using filter paper or other conservation techniques associated with the present culture approach will allow for the accurate identification of potential candidates for clinical trials for the treatment of leishmaniasis.

\section{ACKNOWLEDGMENTS}

To the nursing technicians Marlete Silva Santos and Rosiene Santos Passos, for their contribution to patient care and follow-up procedures, as well as their cooperation in the packaging of samples, and to Renata Ribeiro de Sousa, for processing the samples for the PCR assays.

\section{REFERENCES}

Allahverdiyev AM, Uzun S, Bagirova M, Durdu M, Memisoglu HR 2004. A sensitive new microculture method for diagnosis of cutaneous leishmaniasis. Am J Trop Med Hyg 70: 294-297.

Alvar J, Yactayo S, Bern C 2006. Leishmaniasis and poverty. Trends Parasitol 22: 552-557.
Arévalo J, Ramirez L, Adaui V, Zimic M, Tulliano G, MirandaVerastegui C, Lazo M, Loayza-Muro R, De Doncker S, Maurer A, Chappuis F, Dujardin JC, Llanos-Cuentas A 2007. Influence of Leishmania (Viannia) species on the response to antimonial treatment in patients with American tegumentary leishmaniasis. J Infect Dis 195: 1846-1851.

Bassam BJ, Caetano-Anolles G, Gresshoff PM 1991. Fast and sensitive silver staining of DNA in polyacrylamide gels. Anal Biochem 196: 80-83.

Bensoussan E, Nasereddin A, Jonas F, Schnur LF, Jaffe CL 2006. Comparison of PCR assays for diagnosis of cutaneous leishmaniasis. J Clin Microbiol 44: 1435-1439.

Boggild AK, Miranda-Verastegui C, Espinosa D, Arévalo J, Adaui V, Tulliano G, Llanos-Cuentas A, Low DE 2007. Evaluation of a microculture method for isolation of Leishmania parasites from cutaneous lesions of patients in Peru. J Clin Microbiol 45: 3680-3684.

Boggild AK, Miranda-Verastegui C, Espinosa D, Arévalo J, Martinez-Medina D, Llanos-Cuentas A, Low DE 2008. Optimization of microculture and evaluation of miniculture for the isolation of Leishmania parasites from cutaneous lesions in Peru. Am J Trop Med Hyg 79: 847-852.

Castilho TM, Shaw JJ, Floeter-Winter LM 2003. New PCR assay using glucose-6-phosphate dehydrogenase for identification of Leishmania species. J Clin Microbiol 41: 540-546.

Coggon D, Martyn C, Palmer KT, Evanoff B 2005. Assessing case definitions in the absence of a diagnostic gold standard. Int J Epidemiol 34: 949-952.

Cuba Cuba CA, Netto EM, Costa JL, Barreto AC, Marsden PD 1986a. In vitro culture as a practical tool for the diagnosis and primary isolation of Leishmania braziliensis braziliensis. 2. Studies in patients in endemic areas. Rev Inst Med Trop Sao Paulo 28: 317-324.

Cuba Cuba CA, Netto EM, Marsden PD, Rosa AC, Llanos-Cuentas EA, Costa JL 1986b. Cultivation of Leishmania braziliensis braziliensis from skin ulcers in man under field conditions. Trans R Soc Trop Med Hyg 80: 456-457.

De Assis TSM, Caligiorne RB, Romero GAS, Rabello A 2008. Detection of Leishmania $\mathrm{kDNA}$ in human serum samples for the diagnosis of visceral leishmaniasis. Trans $R$ Soc Trop Med Hyg 103: 540-544.

Degrave W, Fernandes O, Campbell D, Bozza M, Lopes U 1994a. Use of molecular probes and PCR for detection and typing of Leishmania - a mini-review. Mem Inst Oswaldo Cruz 89: 463-469.

Degrave W, Fernandes O, Thieman O, Wincker P, Britto C, Cardoso A, Pereira JB, Bozza M, Lopes U, Morel C 1994b. Detection of Trypanosoma cruzi and Leishmania using the polimerase chain reaction. Mem Inst Oswaldo Cruz 89: 367-368.

Gomes AH, Armelin IM, Menon SZ, Pereira-Chioccola VL 2008. Leishmania (V.) braziliensis: detection by PCR in biopsies from patients with cutaneous leishmaniasis. Exp Parasitol 119: 319-324.

Grimaldi G Jr, Tesh RB 1993. Leishmaniases of the New World: current concepts and implications for future research. Clin Microbiol Rev 6: $230-250$.

Luz ZM, Silva AR, Silva Fde O, Caligiorne RB, Oliveira E, Rabello A 2009. Lesion aspirate culture for the diagnosis and isolation of Leishmania spp from patients with cutaneous leishmaniasis. Mem Inst Oswaldo Cruz 104: 62-66.

Marques MJ, Volpini AC, Genaro O, Mayrink W, Romanha AJ 2001. Simple form of clinical sample preservation and Leishmania DNA extraction from human lesions for diagnosis of Ameri- 
can cutaneous leishmaniasis via polymerase chain reaction. $\mathrm{Am}$ J Trop Med Hyg 65: 902-906.

Marzochi MC, Teixeira PC, Marzochi KB, da Conceição NF, Coutinho W, de Brito DB 1993. Vacuum aspiratory puncture system for Leishmania culturing, isolation and transport. Preliminary report. Rev Inst Med Trop Sao Paulo 35: 301-303.

Murray HW, Berman JD, Davies CR, Saravia NG 2005. Advances in leishmaniasis. Lancet 366: 1561-1577.

Pirmez C, da Silva TV, Paes-Oliveira NM, da Cruz AM, Gonçalvesda-Costa SC, Catanho M, Degrave W, Fernandes O 1999. Use of PCR in diagnosis of human American tegumentary leishmaniasis in Rio de Janeiro, Brazil. J Clin Microbiol 37: 1819-1823.

Reed SG, Badaro R, Masur H, Carvalho EM, Lorenço R, Lisboa A, Teixeira R, Johnson WD Jr, Jones TC 1986. Selection of a skin test antigen for American visceral leishmaniasis. Am J Trop Med Hyg 35: 79-85.

Reis LC, Brito ME, Almeida EL, Felix SM, Medeiros AC, Silva CJ, Pereira VR 2008. Clinical, epidemiological and laboratory aspects of patients with American cutaneous leishmaniasis in the state of Pernambuco. Rev Soc Bras Med Trop 41: 439-443.

Reithinger R, Dujardin JC, Louzir H, Pirmez C, Alexander B, Brooker S 2007. Cutaneous leishmaniasis. Lancet Infect Dis 7: 581-596.

Romero GA, Guerra MV, Paes MG, Cupolilo E, Toaldo CB, Macêdo VO, Fernandes O 2001a. Sensitivity of the polymerase chain reaction for the diagnosis of cutaneous leishmaniasis due to Leishmania (Viannia) guyanensis. Acta Trop 79: 225-229.

Romero GA, Guerra MV, Paes MG, Macêdo VO 2001b. Comparison of cutaneous leishmaniasis due to Leishmania (Viannia) braziliensis and $L$. $(V$.$) guyanensis in Brazil: therapeutic response to$ meglumine antimoniate. Am J Trop Med Hyg 65: 456-465.

Romero GA, Noronha EF, Pirmez C, Pires FE, Fernandes O, Nehme NS, Cupolillo E, Firoozmand L, da Graça GC, Volpini A, Santos SL, Romanha AJ 2009. Sensitivity and reproducibility of a PCR assay for Leishmania detection using skin biopsy imprints on filter paper. Acta Trop 109: 74-77.

Romero GA, Sampaio RN, Macedo V, Marsden PD 1999a. Sensitivity of lymph node aspiration in localized cutaneous leishmania- sis due to Leishmania (Viannia) braziliensis. Mem Inst Oswaldo Cruz 94: 509-511.

Romero GA, Sampaio RN, Macedo VO, Marsden PD 1999b. Sensitivity of a vacuum aspiratory culture technique for diagnosis of localized cutaneous leishmaniasis in an endemic area of Leishmania (Viannia) braziliensis transmission. Mem Inst Oswaldo Cruz 94: 505-508.

Romero GA, Vinitius De Farias GM, Gomes PM, Macêdo VO 2001c. Comparison of cutaneous leishmaniasis due to Leishmania ( Viannia) braziliensis and $L$. (V.) guyanensis in Brazil: clinical findings and diagnostic approach. Clin Infect Dis 32: 1304-1312.

Rosa AC, Cuba CC, Vexenat A, Barreto AC, Marsden PD 1988. Predominance of Leishmania braziliensis braziliensis in the regions of Tres Bracos and Corte de Pedra, Bahia, Brazil. Trans $R$ Soc Trop Med Hyg 82: 409-410.

Safaei A, Motazedian MH, Vasei M 2002. Polymerase chain reaction for diagnosis of cutaneous leishmaniasis in histologically positive, suspicious and negative skin biopsies. Dermatology 205: 18-24.

Sambrook J, Russel DW 2001. Molecular cloning: a laboratory manual, 3rd ed., Cold Spring Harbor Laboratory, NY, 999 pp.

Schallig HD, Oskam L 2002. Molecular biological applications in the diagnosis and control of leishmaniasis and parasite identification. Trop Med Int Health 7: 641-651.

Sokal JE 1975. Measurement of delayed skin-test responses. N Engl J Med 293: 501-502.

Tojal da Silva AC, Cupolillo E, Volpini AC, Almeida R, Romero GA 2006. Species diversity causing human cutaneous leishmaniasis in Rio Branco, state of Acre, Brazil. Trop Med Int Health 11: 1388-1398.

Volpini AC, Passos VMA, Oliveira GC, Romanha AJ 2004. PCRRFLP to identify Leishmania (Viannia) braziliensis and L. (Leishmania) amazonensis causing American cutaneous leishmaniasis. Acta Trop 90: 31-37.

Weigle KA, Labrada LA, Lozano C, Santrich C, Barker DC 2002. PCR-based diagnosis of acute and chronic cutaneous leishmaniasis caused by Leishmania (Viannia). J Clin Microbiol 40: 601-606. 